47 Endra Yustin : The Combination of Platelet Rich Plasma with Skin Needling or Subcision for Postacne Scaring

\title{
The Combination of Platelet Rich Plasma with Skin Needling or Subcision for Postacne Scaring: a Serial Case Study
}

\author{
E. Yustin, A. Kusumawardani, S. Widhiati, I. Julianto \\ Department of Dermatology and Venereology Faculty of Medicine Universitas Sebelas \\ Maret/ General Hospital Dr. Moewardi. \\ *Corresponding author: suci_riza@yahoo.com
}

\begin{abstract}
Scaring in acne remains a common problem and became therapeutic challenges for the clinician. Several modalities were introduced, and combination therapies are required to achieve satisfactory results. Skin needling and subcision reported as effective methods for treating acne scar. The skin needling technique involves puncturing the skin multiple times with a small needle to induce collagen growth, while subcision worked by tunneling the connective tissue and scattered the fibrosis scar. Platelet-rich plasma (PRP) influences wound-healing by stimulating an intense inflammatory response and growth factors, these increase in the production of extracellular matrix and granulation tissue occurs, with vascular ingrowth, fibroblastic proliferation, and collagen production also accelerated. Three male patients enrolled in the study, with grades 2-3 acne scar. There are three different methods of treatment were used to see the effect of the treatment. The first patient used the combination of topical PRP and skin needling, and the second patient received a combination of PRP injection and subcision, while the third patient obtains combination between topical PRP and skin needling continued with PRP injection and subcision. Our study revealed that PRP and skin needling or subcision is a simple technique and has the potential for improvement of acne scars. Three weeks after treatment, all three patient had smoother facial skin and reduction of acne scar severity. The combination of PRP and skin needling or and subcision is a simple, safe and rapid method for acne scars treatment.
\end{abstract}

\section{Keywords: Platelet Rich Plasma, Skin, Acne, Postacne Scaring}

\section{INTRODUCTION}

Acne is a common condition seen in up to $80 \%$ of people between 11 and 30 years of age and in up to $5 \%$ of older adults. In some patients, the severe inflammatory response results in permanent scars. Despite the widespread use of isotretinoin and other therapies to aggressively treat acne, scarring from this condition remains a common problem presenting significant therapeutic challenges. Scars can involve a textural change in the superficial and deep dermis. Once the scar type has been defined, appropriate and effective treatment protocols can be developed (Fabbrocini et al., 2009).

Several modalities have been advocated to treat acne scarring, including surgical techniques (punch grafts, punch excisions, skin needling or dermaroller, subcision), resurfacing techniques (dermabrasion, ablative laser treatment, chemical peels), non-ablative laser treatment, autologous fat transfer, and injection of dermal fillers (Goodman, 2009; Jacob et al., 
2001). A combination of different modalities is typically required to achieve satisfactory results, and each technique carries different risks and side effects.

Skin needling has been used since 1995 to achieve percutaneous collagen induction (PCI) (Orentreich DS and Orentreich N., 1995). It is an effective method for treating acne scars in grades 2-3 (Goodman and Baron, 2007) and other dermatological lesions. The technique involves puncturing the skin multiple times with a small needle to induce collagen growth. In 1995 Orentreich and Orentreich described 'subcision' as a way of building up connective tissue beneath retracted scars and wrinkles. In 2005 Fernandes, simultaneously and independently, used a similar technique to treat the upper lip by inserting a 15-gauge needle into the skin and then tunneling under the wrinkles in various directions, parallel to the skin surface.

Autologous platelet-rich plasma (PRP) is plasma with a higher concentration of platelets than baseline. No definition exists as to that absolute number required. Generally, they are increased up to 3-5 times (Pietrzak and Eppley,

\section{CASE REPORTS}

Three male patients were enrolled in the study (age range 22-24 years) with acne scars grades 2-3 based on Goodman and Barron classification. Exclusion criteria were: the history of a sporadic but severe form of keloid scarring, diabetes, neuromuscular disease, bleeding disorder, collagen vascular disease, acute or chronic corticosteroid or anticoagulant treatment, the presence of skin cancers, warts, solar keratoses or any skin infection. Written
2005). PRP can be applied externally, added to implanted material (Borzini and Mazzucco, 2009; Pallua et al., 2010) or injected directly into a lesion as a matrix for regeneration (Dallari et al., 2006). Application of autologous PRP has been reported to facilitate woundhealing in several fields of surgery (Dallari et al., 2006; Roussy et al., 2007). Platelet-rich plasma influences wound-healing by stimulating an intense inflammatory response and growth factors (Roussy et al., 2007; Leitner et al., 2006) A significant increase in the production of extracellular matrix and granulation tissue occurs, with vascular ingrowth, fibroblastic proliferation, and collagen production also accelerated (Henderson et al., 2003).

Previous experience treatment for acne scarring using the combination between PRP topically with skin needling or PRP injection with subcision never been reported. We hypothesized that the combination system between PRP and skin needling or subcision would be useful in improving acne scars, with minimal downtime and few side effects.

and signed informed consent was obtained from all participants.

The relevance of combination PRP with skin needling or subcision to post acne scarring remains unclear. Our study was tried to make some modification method treatment to see the effect of the treatment. Method 1: a combination between PRP topically and skin needling; method 2: a combination between PRP injection and subcision; and method 3: a 
combination between method 1 and 2. One hour before treatment all patient were applied with topical anesthesia. Skin needling and subcision in our study used the same procedures that already mentioned, but the PRP sources using some modification from the procedures that

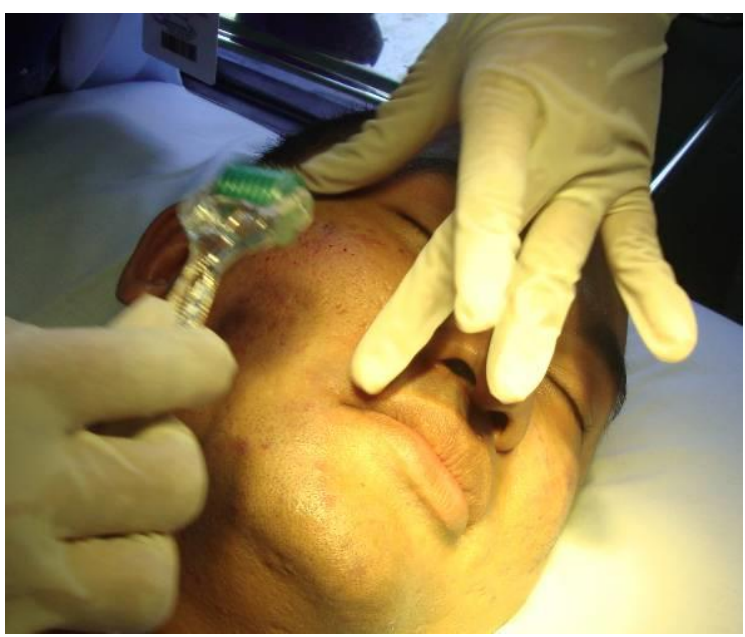

Skin needling with dermaroller kit

Method 1 is a combination between PRP topically and skin needling.

The treatment was then carryout by rolling the needling tool over the area affected as mention in skin needling procedure. As expected, after the treatment, the skin bled for a short time. When the bleeding stopped, a serous ooze formed and then PRP was applied directly to the surface wound. All that methods repeated three times 'sandwich appearance'. After 20-minutes post-treatment, the wound was covered by platelet poor plasma (PPP) mixtures with gentamycin eye ointment. Further wound treatment was not necessary. already mention before (Julianto reported). After treatment, the patient was not allowing the water to soak into the surface of the skin for 24 hours. All the patients only get one season of treatment and will be followed up at week- 3 and month-3.

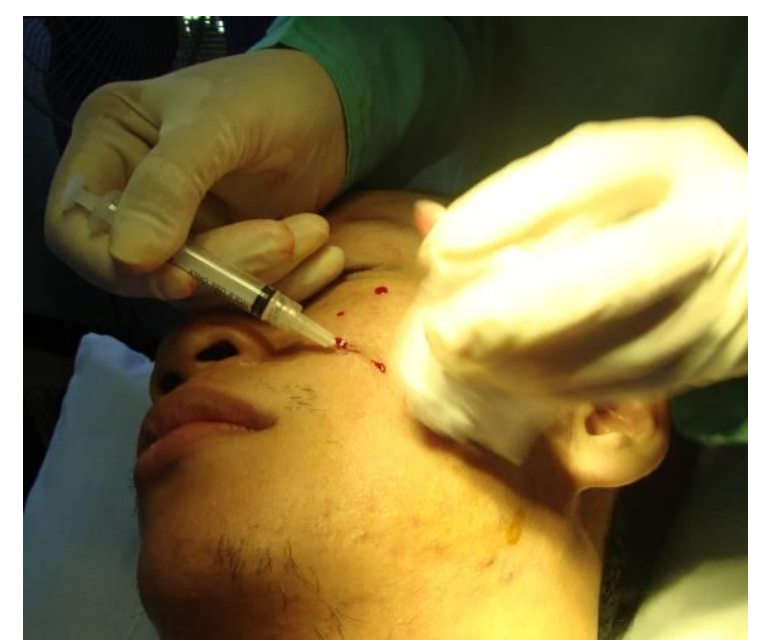

Subcision with a 30 -gauge needle

Method 2 is a combination between PRP injection and subcision.

After subcisions in acne scar, the tunneling is performed sufficiently to almost free the attachments. The instrument is passed sideways in a sweeping action to complete the freeing up of the skin from its base. The depression will be seen, and the procedure is complete. This procedure was followed by injection of PRP in the same place with subcision before $(0,1 \mathrm{ml}$ PRP/one subcision place). After treatment, the wound was covered by PPP mixtures with gentamycin eye ointment. Further wound treatment was not necessary.

Method 3 is a combination between method 1 and 2 . 


\section{Method 3 is a combination between method 1} and 2. Method 1 chosen for acne rolling scar and method 2 for isolated moderate atrophic scars (large).

After each session of treatment, the facial skin appeared reddened and swollen, but patients stated that the redness and swelling disappeared in 2-3 days. No side effect was reported or found. Three weeks after the treatment, all patients had smoother facial skin and a reduction in lesion severity. From this study result, we cannot conclude the superior method for acne scarring treatment from 3 method modification. It needed for further investigation.
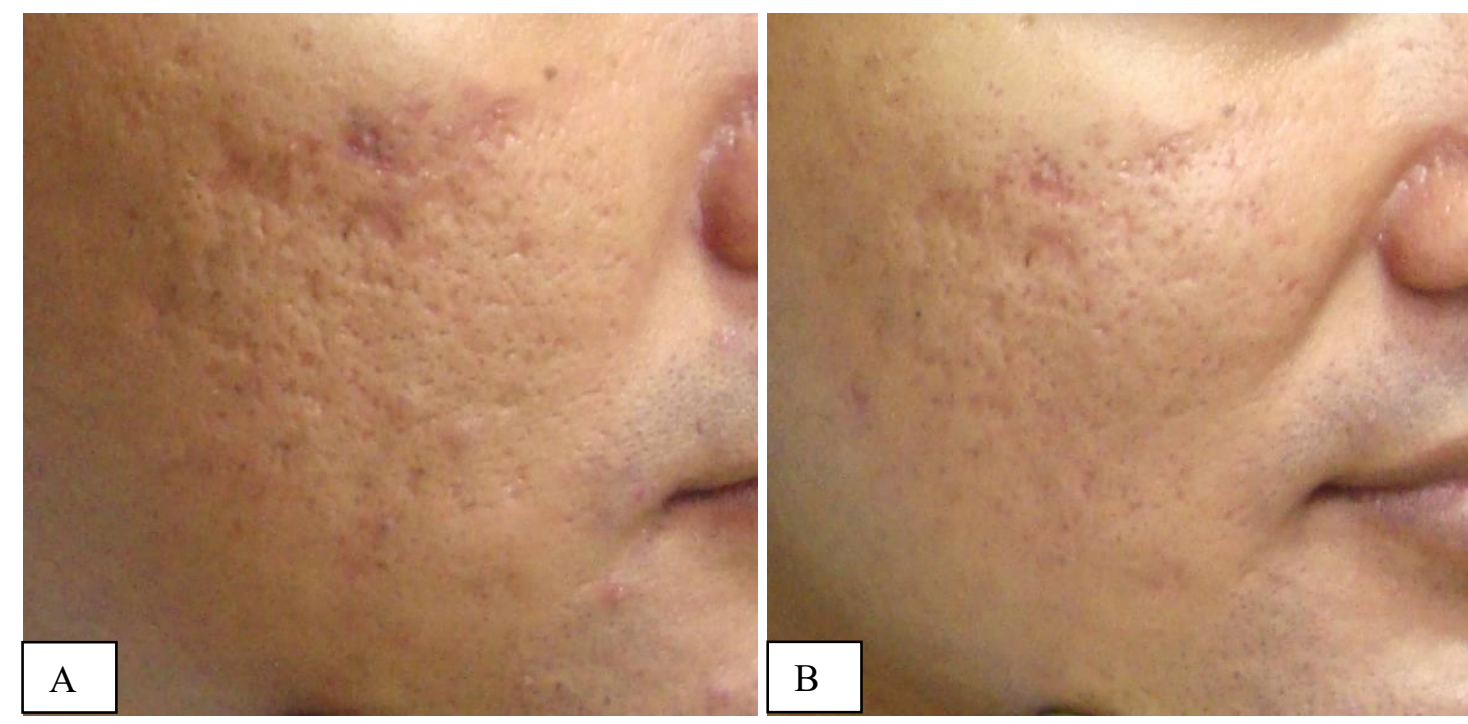

Figure 1. a combination between PRP topically and skin needling

(A) Acne scar before treatment; (B) improvement acne scar 3-weeks after treatment.
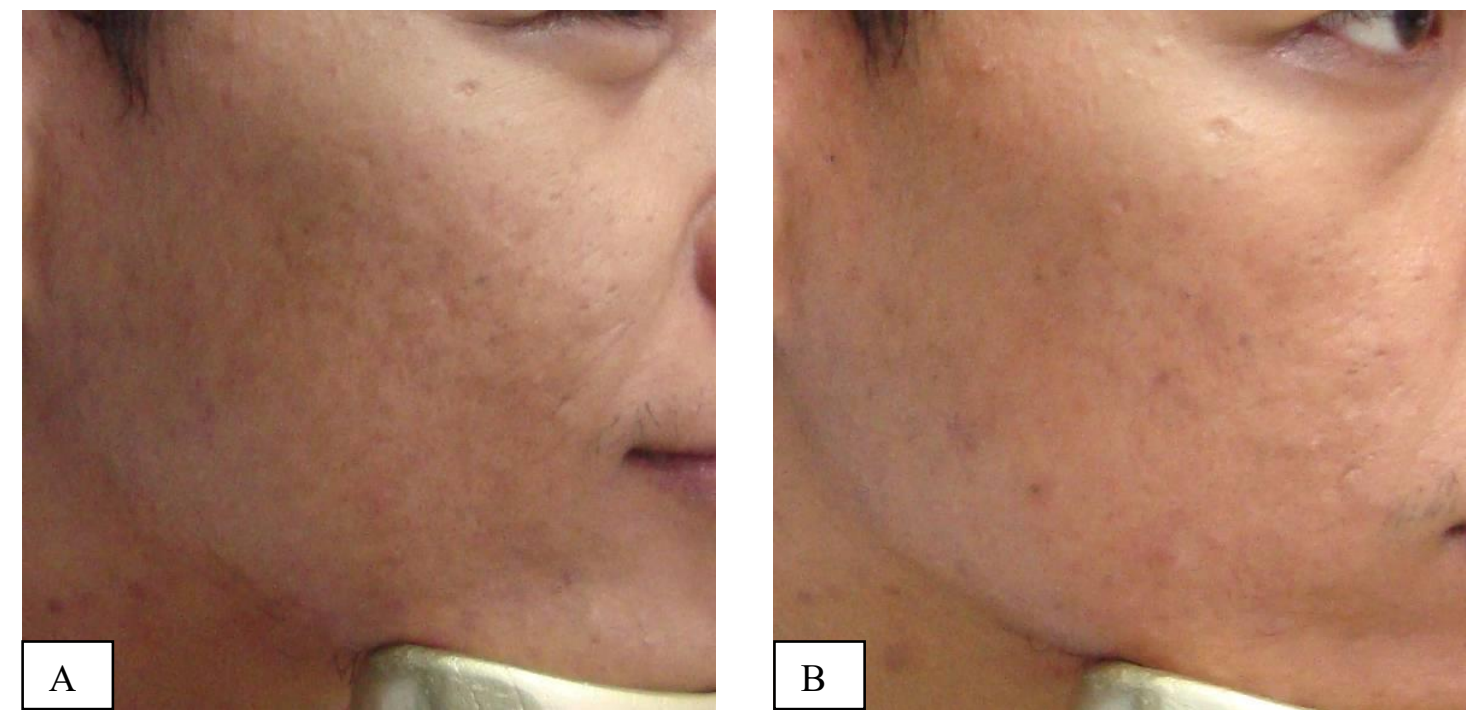

Figure 2. a combination between PRP injection and subcision (A) Acne scar before treatment;

(B) improvement acne scar 3-weeks after treatment. 


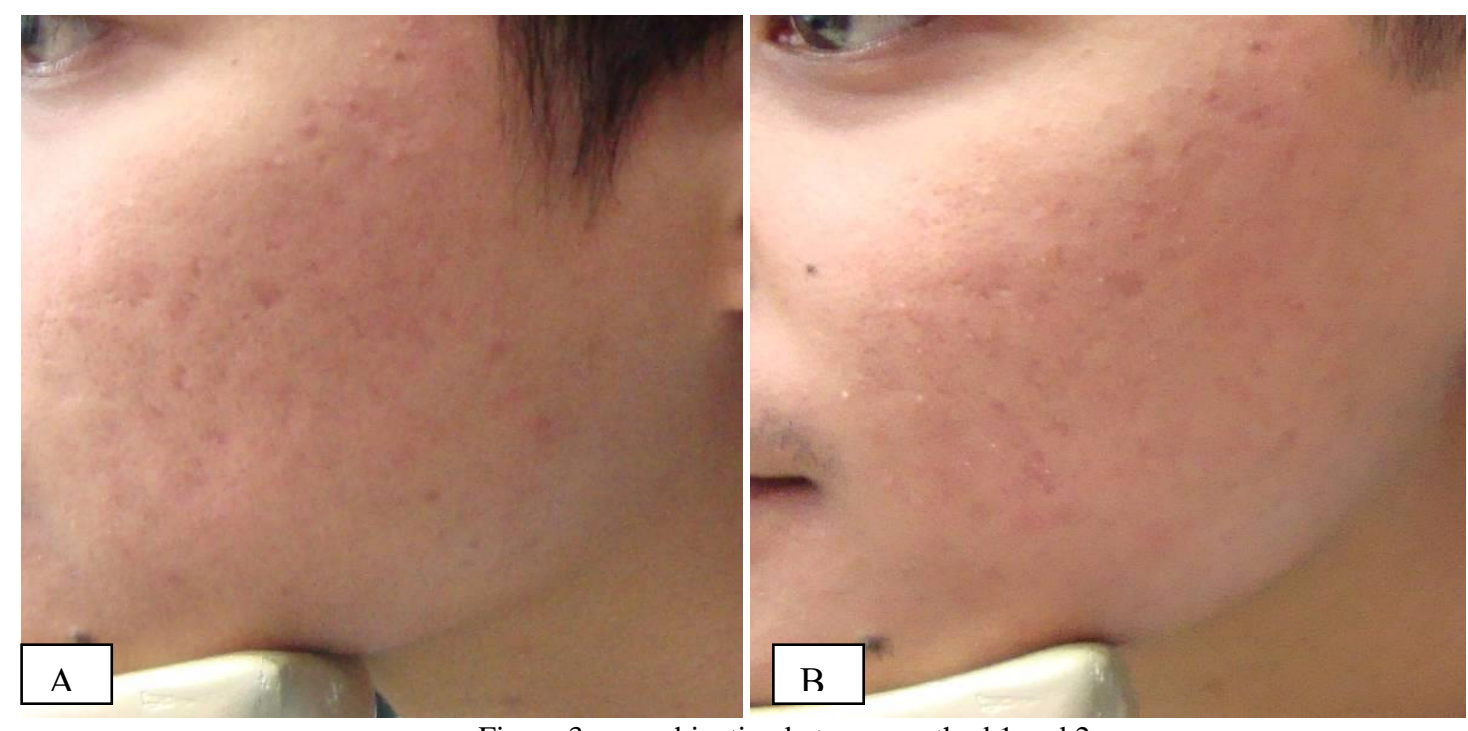

Figure 3. a combination between method 1 and 2

(A) Acne scar before treatment; (B) improvement acne scar 3-weeks after treatment.

\section{DISCUSSION}

When a needle penetrates into the skin, the injury causes localized damage and minor bleeding by rupturing little blood vessels. A completely different picture emerges when thousands of fine needle pricks are placed close to each other. Most authors consider that skin needling induces normal wound healing, developing in three phases (Diegelmann and Evans, 2004). Phase 1, the inflammation phase, starts soon after the injury; platelets release chemotactic factors, which cause invasion of other platelets, neutrophils, and fibroblasts. During proliferation (phase 2), neutrophils are replaced by monocytes that change into macrophages and release several growth factors including platelet-derived growth factor, fibroblast growth factor, and transforming growth factors $\alpha$ and $\beta$, which stimulate the migration and proliferation of fibroblasts. Keratinocytes then become mobile to cover the gap in the basement membrane. They start producing all the components to re-establish the basement membrane with laminin and collagen types IV and VII. A day or two after PCI, the keratinocytes begin to proliferate and release growth factors to promote collagen deposition by the fibroblasts. Tissue remodeling (phase 3 ) continues for months after the injury and it mainly achieved by the fibroblasts: collagen type III is laid down in the upper dermis and gradually replaced by collagen type I over a period of a year or longer.

Skin needling is rolling of the dermaroller kit over scar tissue. It is the exact procedure with vertical subcision since the rolling of the needles over the scar will vertically cut the fibrous septae and release the skin of its fibromuscular attachment to deeper tissues. This needling help release tension coming from the underlying SMAS (Superficial Musculoaponeurotic System) in facial areas (Deepali, 2012).

Percutaneous Collagen Induction (PCI), stimulating the dermis to produce collagen through the induction of thousands of minuscule wounds on the skin through the use of needles. Each tiny wound goes through the three classic phases of wound healing. The 
needle only penetrates through the epidermis and does not remove it, so the epidermis is only pierced and will rapidly heal in a matter of hours since keratinocytes are highly elastic and flexible. Skin capillaries are ruptured, and so blood cells and serum get into the surrounding tissue. A complex chemical cascade determines the formation of hundreds of miniscule clots and the vascular permeability, the chemoattraction for leucocytes and the recruitment of fibroblasts in the wounded area. Platelets cause clotting and release chemotactic factors like Platelet Derived Growth Factor (PDGF), Transforming Growth Factor (TGF) and Fibroblast Growth Factor (FGF) that initiate an invasion of other platelets, leucocytes, and fibroblasts. Neutrophils act on the "damaged" tissue by removing the debris of the old and damaged collagen and microclots. This reaction is automatic and produces a surge of activity that inevitably leads to the fibroblast being instructed to produce more collagen and more elastin (Fernandes, 2005; Orentreich DS and Orentreich N., 1995).

The re-epithelialization occurs within a few hours after needling and is really due to keratinocyte migration rather than proliferation. When the keratinocytes have joined together, they start producing all the components to reestablish the basement membrane with laminin and collagen types IV and VII. One or days after the injury, the keratinocytes start proliferating and thicken the epidermis. Fibroblasts migrate into the wound and produce collagen, proteoglycans, elastin and other matrix proteins. Initially, after needle damage, the disruption of the blood vessels causes a moderate amount of hypoxia. The low oxygen tension stimulates the fibroblast to produce more TGF, PDGF, and VEGF (Vascular Endothelial Factor). TGF is a powerful chemotactic agent for fibroblasts, which migrate into the wound at about 48 hours after injury and start producing collagen I and III, elastin. Glycosaminoglycans and proteoglycans. Collagen type III is the dominant form of collagen in the early wound healing phase and becomes maximal by 5-7 days after injury. The collagen is laid down on the upper dermis just below the basal layer of the epidermis (Fernandes, 2005; Orentreich DS and Orentreich N., 1995; Gabling et al., 2009).

Skin needling appears to be repeatable and, probably more pertinent appears to be synergistic with other methods such as nonablative lasers, blood transfer, and vascular lasers. Goodman and Baron's practice always supplements the procedure of skin rolling or needling with other procedures, both simultaneously (blood transfer, vascular laser, and subcision for more prominent scars) and sequentially starting one month after the procedure. There is scientific proof that the needling procedure also stimulates revascularization, repigmentation of stretch marks and filling of cutaneous wrinkles.

Subcision is a technique of undermining scars. Subcision of scars appears to work by breaking up the attachments of these contour abnormalities and releasing the surface from the deeper structures results in skin elevation. Blood accumulates under the defect, and its subsequent organization initiates wound healing with consequent formation of connective tissue that augments the depressed scar, leads to long-term correction of the defect, 
although this is usually not complete after the first treatment. Successive treatments appear to produce further improvement. The technique of undermining scars has widely practiced over many years as an adjunct to other treatment (Goodman and Baron, 2007).

After subcision, some bleeding appears to be useful to establish a short-term spacer to keep the tissues from early reattachment, but the later organization of the ecchymosis may be responsible for the laying down of new host collagen. Predictable sequelae such as bruising and swelling are often present for 1 to 2 weeks. A range of responses is seen ranging from partial to excessive. Partial response is usual whereas excess response is seen in $5 \%$ to $10 \%$ and may require patience because original resolution usually occurs, albeit slowly, over months. As a simple technique that appears to produce a long-term correction of contour defects, it deserves to be a first-line treatment for many isolated moderate atrophic scars (Goodman and Baron, 2007).

Some reports have indicated that the application of autologous PRP accelerates the regeneration of tissue defects. At the same time, controlled and scientifically proven clinical studies remain to be published. Up to the present, the relevance of PRP to post-acne scarring remains unclear. PRP has shown to induce an intense inflammatory response manifesting as the production of granulation tissue.

Platelets are anucleate cytoplasmic fragments derived from bone marrow megakaryocytes. They form an intracellular storage pool of proteins vital to wound healing, including platelet-derived growth factor
(PDGF), transforming growth factor $-\beta$ (TGF$\beta)$ (including $\beta 1$ - and $\beta 2$-isomers), and insulinlike growth factor I (IGF-I). The $\alpha$-granules fuse with the platelet cell membrane after activation. At least some secretory proteins are transformed to a bioactive state. The active proteins are then secreted, allowing them to bind to transmembrane receptors of the target cells. Once bound, intracellular signal proteins are activated. These results were in the expression of a gene sequence that directs cellular proliferation, collagen synthesis, osteoid production, and so on (Pietrzak and Eppley, 2005; Roussy et al., 2007; Leitner et al., 2006).

The result of the experiment found that Platelet-rich plasma (PRP) contains growth factors, such as PDGF (AB and $\mathrm{BB}$ ) and TGF$\beta$ (Roussy et al., 2007; Leitner et al., 2006; Gabling et al., 2009). VEGF and interleukin-1 $\beta$ (IL-1ß) (Roussy et al., 2007). All that growth factors were needed in wound healing as mention before.

There is no definitive and straightforward solution for acne scarring, which is a difficult problem to treat. A combination of several treatment procedures may be appropriate, depending on specific patient features. Skin needling, subcision, PRP and all its therapeutic possibilities are now being researched intensively. All that treatment base on wound healing phase in the acute lesion. A combination PRP with skin needling or subcision, which never been reported, will give a synergistic result.

The results of our study, however, confirm that the combination system between PRP and skin needling or subcision is a simple technique 
and can have an immediate effect on the improvement of acne scars. Following the literature, the full result may take 8-12 months of treatment to occur, as the deposition of new collagen takes place slowly (Fernandes and Signorini, 2008). In our study result, at 3-week after the treatment, all patient had smoother facial skin and a reduction in lesion severity. The observation will be continuing to see the

\section{REFERENCES}

Borzini P. and Mazzucco I. Platelet-rich plasma (PRP) and derivatives for topical therapy. What is true from the biologic viewpoint? Science Series. 2007; 2: 272-81.

Dallari D, Fini M., Stagni C, Torricelli P, Nicoli A, et al. In vivo study on the healing of bone defect treated with bone marrow stromal cells, Platelet Rich Plasma, and freeze-dried bone allograft, alone and in combination. J Orthop Res. 2006; 24: 877-88.

Deepali B. Collagen induction therapy with dermaroller: a review. CBMJ. 2012; 01:01:35-37.

Diegelmann RF, Evans MC. Wound healing: an overview of acute, fibrotic and delayed healing. Frontiers in Bioscience. 2004; 9: 282-9.

Fabbrocini G, Fardella N, Monfrecola A, Proietti I, Innocenz D. Acne scarring treatment using skin needling. Clin and Exper Dermatol. 2009; 34: 874-9.

Fernandes D. Minimally invasive percutaneous collagen induction. Oral Maxillofac Surg Clin North Am. 2005; 17: 51-63.

Fernandes D, Signorini M. Combating photoaging with percutaneous collagen best result. We believe that the combination of PRP and skin needling or subcision is a rapid and straightforward method for the safe treatment of acne scars and a suitable procedure for various dermatological conditions.

Further experimental needed with a broader subject, observation and also compared with other methods as a control.

induction. Clin Dermatol. 2008; 26: 1929.

Gaßling VLW, Açil Y, Springer IN, Hubert N, Wiltfang J. Platelet-rich plasma and platelet-rich fibrin in human cell culture. Oral Surg Oral Med Oral Pathol Oral Radiol Endod. 2009; 108: 48-55.

Goodman GJ., and Baron JA. The management of post-acne scarring. Dermatol Surg. 2007; 33:1175-88.

Goodman GJ. Post acne scarring: A review of its pathophysiology and treatment. Dermatol Surg. 2009; 26: 857-71.

Henderson JL, Cupp CL, Ross EV, Shick PC, Keefe MA, Wester DC, et al. The effects of autologous platelet gel on wound healing. Ear Nose Throat J. 2003; 82: 598-02.

Kadunc BV, Trindade de Almeida AR. Surgical treatment of facial acne scars based on morphologic classification: a Brazilian experience. Dermatol Surg. 2003; 29:1200-9.

Jacob CI, Dover JS, Kaminer MS. Acne scarring: A classification system and review of treatment options. J Am Acad Dermatol. 2001; 45:109-17. 
Leitner GC, Gruber R, Neumuller J, Wagner A, Kloimstein P, et al. Platelet content and growth factors released in platelet-rich plasma: a comparison of four different systems. Vox Sanguinis. 2006; 91: 1359.

Orentreich DS, Orentreich N. Subcutaneous incisionless (subcision) surgery for the correction of depressed scars and wrinkles. Dermatol Surg. 1995; 21: 5439.
Pallua N, Wolter T, Markowicz M. Platelet-rich plasma in burns. Burns. 2010; 36:4- 8 .

Pietrzak WS, Eppley BL. Platelet-rich plasma: biology and new technology. J Craniofac Surg. 2005; 16(6):1043-54.

Roussy Y, Bertrand MP, Gagnon DG. Activation of human platelet rich plasmas: effect on growth factors release, cell division and in vivo bone formation. Clin Oral Impl res. 2007; 18: 639-48. 